\title{
Detection of intracardiac thrombi in a case of cardiomyopathy by two dimensional echocardiography
}

\author{
MIKIO ARITA, YUJI UENO, „ YOSHIAKI MASUYAMA \\ From the Division of Cardiology, Wakayama National Hospital, Wakayama, Fapan
}

SUMMARY A case of cardiomyopathy with systemic emboli is reported. Two dimensional and M-mode echocardiography showed abnormal intracavitary echoes. After further systemic emboli, echocardiograms showed the complete disappearance of the abnormal echoes found previously in the apex, which suggested that the emboli resulted from detachment of the left ventricular mural thrombi.

Intracardiac mural thrombi are frequently associated with ventricular hypokinesia such as occurs in myocardial infarction and cardiomyopathy. ${ }^{12}$ Most of these thrombi are discovered during necropsy and are difficult to detect clinically. We describe the two dimensional and $M$-mode echocardiographic features of left ventricular thrombi which were found in a case of congestive cardiomyopathy, and which disappeared completely after they became detached as systemic emboli.

\section{Case report}

An 8-year-old girl was admitted to Wakayama National Hospital in February 1980, with orthopnoea and right sided weakness. Two weeks before being admitted she had had an influenza type illness and had experienced increasing cough and shortness of breath, which seemed worse at night. Previously, when 3 years old, she had had an operation for persistent ductus arteriosus. Her elder brother had died of malignant hypertension at 6 years of age.

On examination her heart rate was 150 and the respiratory rate 45 per minute. The first and second heart sounds were normal. A third heart sound was noted at the apex. Respiration was laboured, with tachypnoea and orthopnoea. The liver was palpable 3 $\mathrm{cm}$ below the right costal margin. There was pitting oedema of both lower legs and feet. There was no pulsation in both radial and the right femoral arteries; there was only a weak pulsation in the left femoral and

^Present address: Division of Cardiology, Department of Medicine, Wakayama Medical College, Wakayama, Japan. both dorsalis pedis arteries. Because of the pulse deficits the blood pressure could not be recorded. Examination of the nervous system showed a right hemiplegia, a right facial palsy, and motor aphasia.

The laboratory findings were: haematocrit $38 \%$; the white blood cell count 22800 , with $72 \%$ neutrophils and $6 \%$ band forms, $17 \%$ lymphocytes, and $5 \%$ monocytes, blood chemistry within normal limits, erythrocyte sedimentation rate $21 \mathrm{~mm}$ in one hour, C-reactive protein was $4+$, viral antibody titres all normal. A chest $x$-ray film showed cardiomegaly and pulmonary congestion, and an electrocardiogram showed sinus tachycardia at a rate of 150 , with deep $Q$ waves in II, III, aVF, and V2-5. The girl's congestive heart failure was treated with digitalis, diuretics, and bed rest. After five days in hospital her condition improved and the radial pulses returned to normal.

On the sixth day, however, systemic emboli occurred. The patient suddenly became unresponsive to stimuli, with conjugated deviation of the eyes to the left. The right radial artery became impalpable once more. An echocardiogram showed dilatation of the left ventricle with hypokinesia and thinning of the interventricular septum. The amplitude of movement of the mitral valve was decreased and its closure was prolonged and interrupted ("shoulder"). The left atrium, right ventricle, and aortic root were all normal.

A two dimensional echocardiogram showed abnormal echoes in the apex (Fig. 1). These abnormal echoes moved during cardiac contraction and were thought to be thrombi. They were not at first detected on M-mode echocardiography but as the transducer was rotated from the base toward the apex of the left ventricle, additional intracavitary densities were seen 

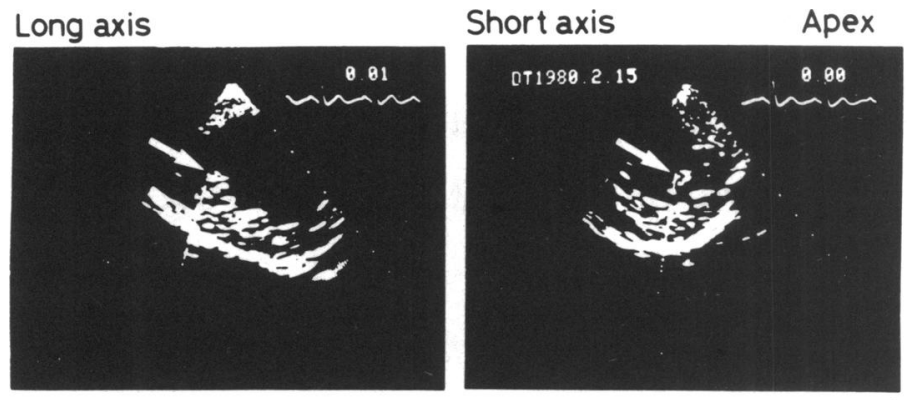

Fig. 1 Two dimensional echocardiograms before (upper) and after (lower) systemic embolisation. Mural thrombus (arrows) is shown in the apex, which disappeared completely after systemic embolisation.
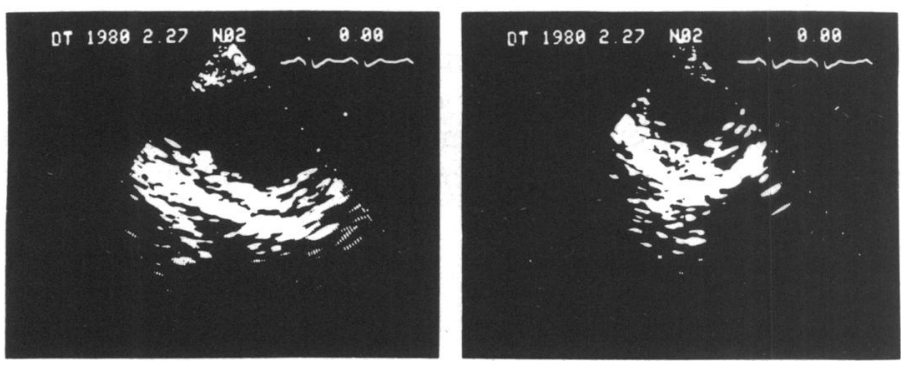

in the apical area adjacent to the posterior wall (Fig. 2). After further systemic emboli occurred (cerebral, right radial) the $M$-mode and the two dimensional echocardiograms showed complete disappearance of these abnormal apical echoes (Fig. 1, 2). A CT scan
Fig. 2 M-mode echocardiograms before (upper) and after (lower) systemic emboli had occurred, showing a mass of echoes (arrow) in the apical area which disappeared.
Feb.16,1980

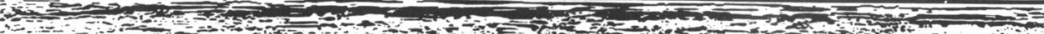
and

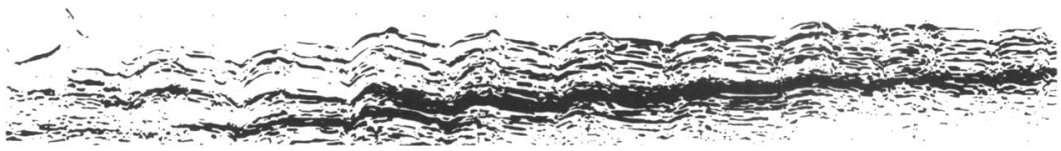

on the 24th hospital day indicated a low density in the areas of the lentriculo-striate artery. This was made more distinct by the contrast method. Though motor aphasia was improved by subsequent treatment, right hemiplegia persisted. 


\section{Discussion}

Left ventricular thrombi commonly occur in myocardial infarction and cardiomyopathy. Spodick and Littmann $^{3}$ found that over $50 \%$ of cases of cardiomyopathy were found at necropsy to have thrombi. Though thromboembolism occurs more frequently in older patients, Harris et al. ${ }^{4}$ reported emboli in four of 10 children with cardiomyopathy.

In our case, cardiac catheterisation was not performed because left ventriculography is dangerous in cases with mural thrombi and this is especially true in children. Our patient had no past history suggesting myocardial infarction or the mucocutaneous lymph node syndrome. Therefore, congestive cardiomyopathy was diagnosed. After systemic emboli had occurred, abnormal intraventricular echoes suggested mural thrombi and these disappeared when further emboli occurred.

Thrombi often occur in the left atrium and the value of echocardiography in diagnosing these is well known, ${ }^{56}$ but the reliability of echocardiography in detecting left ventricular thrombi has not been fully assessed. Horgan et al. ${ }^{7}$ reported a left ventricular thrombus detected by $\mathbf{M}$-mode echocardiography. Subsequently, Kramer et al. ${ }^{8}$ described a patient with alcoholic cardiomyopathy with left ventricular mural thrombi.

Horgan et al. ${ }^{7}$ stated that a thrombus must be both large and organised in order to be detected by echocardiography. Though some authors have reported that $\mathbf{M}$-mode echocardiography is a useful technique in detecting intracavitary thrombi, it is less useful in demonstrating mural thrombi. Ports et al. ${ }^{9}$ reported that both intracavitary and intramural left ventricular tumour masses were detected with $M$-mode and two dimensional echocardiography. $M$-mode echocardiography, however, did not show a ventricular thrombus in all instances. ${ }^{9}$ Two dimensional echocardiography easily identified the large and inhomogeneous left ventricular thrombi, but it could not always clearly show smaller mural thrombi.

In our case, intraventricular mural thrombi were directly visualised and their location, size, and subsequent movement were detected with two dimensional echocardiography, which was therefore valuable.

We wish to thank Drs Kozo Takenaka and Shinji Mikita for their valuable advice in this case.

\section{References}

1 Hellerstein HK, Martin JW. Incidence of thromboembolic lesions accompanying myocardial infarction. $\mathrm{Am}$ Heart f 1947; 33: 443-52.

2 Stapleton JF, Segal JP, Harvey WP. Clinical pathways of cardiomyopathy. Circ Res 1974 ; 34 \& 35, suppl II: 168 78.

3 Spodick DH, Littmann D. Idiopathic myocardial hypertrophy. Am $\mathcal{F}$ Cardiol 1958; 1: 610-23.

4 Harris LC, Rodin AE, Nghiem QX. Idiopathic, nonobstructive cardiomyopathy in children. Am $\mathcal{F}$ Cardiol 1968; 21: 153-65.

5 Wolfe SB, Popp RL, Feigenbaum H. Diagnosis of atrial tumors by ultrasound. Circulation 1969; 39: 615-22.

6 Popp RL, Harrison DC. Ultrasound for the diagnosis of atrial tumor. Ann Intern Med 1969; 71: 785-7.

7 Horgan JH, Shiel FO, Goodman AC. Demonstration of left ventricular thrombus by conventional echocardiography. $F C U$ 1976; 4: 287-8.

8 Kramer NE, Rathod R, Chawla KK, Patel R, Towne WD. Echocardiographic diagnosis of left ventricular mural thrombi occurring in cardiomyopathy. Am Hean $\mathcal{f}$ 1978; 96: 381-3.

9 Ports TA, Cogan J, Schiller NB, Rapaport E. Echocardiography of left ventricular masses. Circulation 1978; 58: $528-36$.

Requests for reprints to Dr M Arita, Division of Cardiology, Department of Medicine, Wakayama Medical College, 1. 7-Bancho, Wakayama City, 640 Japan. 